\section{Процессно-ориентированный подход к внедрению корпоративных информационных систем в лесном комплексе}

\author{
М. В. Федоров ${ }^{1}$ \\ Петрозаводский государственный университет
}

\begin{abstract}
АННОТАЦИЯ
В статье описываются возможности информационных систем с точки зрения применения их в управлении предприятиями лесного комплекса. А также излагаются особенности, преимущества и недостатки функционального и процессно-ориентированного подходов к внедрению корпоративных информационных систем.
\end{abstract}

Ключевые слова: управление производством, корпоративные информационные системы, бизнеспрочессы, функииональный подход, прочессноориентированный подход.

\section{SUMMARY}

The article covers the potential of information systems used in enterprise management of wood-processing industry. It also focuses on features, advantages and disadvantages of functional and process-oriented approaches to introduction of corporative information systems.

Keywords: production management, corporative information systems, business processes, functional approach, process orientation approach.

Отечественная и зарубежная наука убедительно доказывает многогранное значение леса, как для окружающей нас среды, так и для развития человечества в целом. Леса обеспечивают не только экологическое равновесие, но и устойчивое экономическое и социальное развитие целых регионов и наций. В последние годы вопросы пользования лесами без нанесения невосполнимого ущерба естественным природным комплексам приобретают все большую актуальность.

Эффективные технологические и организационные решения, позволяющие на соответствующем современным требованиям уровне обеспечить ведение неистощимого лесопользования, предъявляют гораздо более высокие требования к качеству и оперативности реагирования на изменяющуюся ситуацию. В связи с этим одной из основных проблем, стоящих в настоящее время перед предприятиями лесного комплекса, является обеспечение эффективного управления.

С другой стороны, экономическая ситуация в России и складывающиеся рыночные отношения требуют

\footnotetext{
${ }^{1}$ Автор-аспирант кафедры тяговых машин (C) М. В. Фёдоров, 2003
}

очень серьезного пересмотра принципов и механизмов управления на уровне каждого предприятия.

Одним из основных способов повышения эффективности управления производством является автоматизация управления, связанная с внедрением новых информационных технологий и методов принятия управленческих решений.

Применение информационных систем в лесном комплексе позволит решить наиболее актуальные проблемы, стоящие перед руководителями предприятий:

$>$ хозяйственный учет;

$>$ оперативный учет;

$>$ планирование производства;

$>$ планирование реализации продукции;

$>$ планирование технического обслуживания и ремонта оборудования.

Кроме того, информационные системы позволяют усовершенствовать многие внутренние процессы, снизить себестоимость продукции, производить ее быстрее и более высокого качества, чем раньше, вскрыть новые резервы, качественно улучшить бизнес предприятия и обеспечить ему устойчивую позицию в рыночной конкуренции. Пример таких систем - корпоративные информационные системы.

В настоящий момент на рынке внедрения корпоративных систем сложилась непростая ситуация из-за того, что предприятия при внедрении систем такого уровня делают упор на автоматизацию отдельных функций, следуя стратегии функционально ориентированного внедрения. Такой подход значительно упрощает задачу на этапах планирования и разработки систем, но не дает ожидаемых результатов на этапах внедрения и промышленной эксплуатации, так как ориентируется на автоматизацию конкретных функций, практически не учитывая при этом сложные межмодульные связи и функционирование системы в целом.

При функционально ориентированном внедрении на каждом этапе проекта происходит постепенное наращивание системы дополнительными модулями, которые расширяют ее функционал. Для реализации такого подхода, как правило, не требуется взгляд на систему как на единое целое.

Подключаемые функциональные модули могут слабо взаимодействовать с уже внедренными на предыдущих этапах, что приводит к необходимости возврата на предыдущие этапы внедрения, многократному перепроектированию системы и, как следствие, к существенному увеличению сроков и затрат на внедрение.

В результате информационное пространство корпоративной системы на конечном этапе внедрения выглядит не как единая материя, а как некая совокупность лоскутов, не лучшим образом сшитых между собой. Такая система не имеет больших преимуществ по отношению к ситуации, имевшей место до ее внедрения. 
Яркий пример ошибочности подобного подхода история внедрения АСУ в Советском Союзе и в других развитых странах. Уже в начале 80 -х годов Госплан СССР фиксировал отрицательный эффект от внедрения АСУ по отдельным предприятиям и отраслям (-18\%). Иными словами, программа АСУ, призванная стать информационной поддержкой экономики страны, стала тормозом не только в экономике, но и в идеологии развития управления.

Другим недостатком функционально ориентированного подхода при внедрении корпоративных систем является то, что каждый конечный исполнитель заинтересован только в хорошем выполнении закрепленных за ним функций (для отчетов перед непосредственным руководством) и не беспокоится, как результаты его работы влияют на функционирование предприятия в целом и на достижение предприятием конечных целей.

Внедрение корпоративных систем и их последующая эффективная работа возможны лишь при наличии четко сформулированных правил взаимодействия отдельных объектов этой системы, для их выработки нужна информация, которая может быть получена лишь при использовании процессноориентированного подхода как на этапе проработки всего проекта, так и на этапе внедрения. Чтобы говорить о системе, необходимо иметь четкое представление о цели и смысле ее существования. Основой развития системы является процесс, направленный на достижение конкретной цели.

Основными этапами реализации процессноориентированного подхода являются формулирование глобальной цели предприятия и описание правил ее достижения. Эти правила должны содержать описание отношений между структурными подразделениями компании, а правила управления выходами получение результатов отработки конкретных функций, описание информационных и коммуникационных технологий.

Первый шаг на пути процессно-ориентированного подхода - выделение целей и конечных продуктов компании, выстраивание бизнес-процессов по продуктовым линиям и увязка их в сквозные процессы, называемые сценариями.

Жизненный цикл проекта по внедрению корпоративных систем включает несколько стадий.

1. Анализ и спецификация (подготовка проекта) планирование и организация работ по проекту, определение рамок проекта, определение и принятие языка моделирования и анализ процессов, введение стандартов проектирования, анализ отчетности, построение модели системы.

2. Спецификация требований (разработка проекта) - построение системного прототипа, отображающего все процессы и процедуры в компании в принятых нормативных документах и стандартах.
3. Внедрение и тестирование (реализация) - данная стадия предваряет запуск системы в производство: установка на рабочих местах и документирование системы, обучение сотрудников, перенос данных из существующих систем в разработанную, передача системы в промышленную эксплуатацию.

4. Контроль качества и тренинг осуществляются в течение всего жизненного цикла системы.

5. Внесение изменений и поддержка - сбор замечаний от пользователей и разработка процедур по внесению изменений в систему.

6. Непрерывное совершенствование и оптимизация осуществляются в течение всего жизненного цикла системы.

Рассмотрим преимущества использования процессноориентированного подхода на каждой стадии проекта по внедрению корпоративной системы.

Подготовка проекта. На этой стадии строится модель системы, которая позволяет определить рамки проекта, получить представление о конкретных объектах, а также описать правила поведения системы в целом. Процессно-ориентированный подход на стадии подготовки проекта позволяет объединить описания организационной структуры, структуры данных и функций в единую модель взаимосвязанных бизнеспроцессов, что, в свою очередь, дает возможность получать представление о работе организации с различных точек зрения:

$>$ организационной - доступ сотрудников подразделений к функциям и элементам данных бизнес-процессов системы;

$>$ функциональной - элементом какого бизнеспроцесса является та или иная функция, какое событие является первопричиной выполнения функции, какие документы являются входящими, а какие исходящими, кто отвечает за выполнение функции;

$>$ информационной - какие данные необходимы на каждом этапе бизнес-процессов для выполнения той или иной функции;

$>$ процессной - как функции объединены в процесс, какие данные необходимо передавать от функции к функции в рамках процесса, как участники процесса (сотрудники) взаимодействуют.

По своей сути корпоративные системы являются интегрированными, и при их внедрении ранее определенные функции отделов предприятия часто пересекаются. В силу этого при внедрении системы происходит перераспределение функций между представителями различных подразделений. Процессноориентированный подход позволяет учесть эту особенность корпоративных систем уже на этапе планирования (моделирования).

Разработка и реализация. На этих стадиях происходит постепенное погружение в бизнес-процессы предприятия. Такая стратегия носит название "Стратегия итеративной разработки" и характеризуется наличием 
нескольких итераций, на каждой выполняются шаги по разработке и реализации системы, удовлетворяющей набору требований к проекту в целом.

Раньше стадии разработки и реализации были, как правило, разделены, но в соответствии с предлагаемой стратегией они протекают одновременно. Такой подход дает возможность непосредственного использования знаний стадии разработки при реализации проекта, а также знаний, полученных при реализации, для изменения модели системы (модели-прототипа). Значит, на каждой последующей итерации по разработке и реализации можно использовать более адаптированную для данного предприятия модельпрототип системы и проводить работы по проекту более качественно.

Стратегия итеративной разработки в совокупности с процессно-ориентированным подходом характеризуется тем, что на каждом этапе-итерации внедрения рассматривается единичный сквозной процесс, характеризующийся определенными условиями входа и выхода (результатом). При этом целью на каждом этапе-итерации будет охватывание все большего числа бизнес-процессов предприятия, в отличие от функционально ориентированных стратегий разработки, где на каждом этапе отрабатываются конкретные функции отдельных подразделений.

При таком подходе на каждом этапе разработки и внедрения особое внимание уделяется именно механизмам взаимодействия структурных подразделений, нацеленных на получение конечного продукта, а не на фиксирование разработки на конкретных функциях. Это позволяет внедрять систему не помодульно, а попроцессно, т. е. на каждом этапе внедрения автоматизировать выделенный сквозной процесс. В последующих итерациях разработки происходит подключение новых процессов к системе - это либо разработка новых модулей, либо развитие функционала существующих.

Если на этапе подготовки проекта было выделено несколько продуктов, которые характеризуются определенными бизнес-процессами, объединяющими в единый процесс подразделения (например, добыча, производство, вспомогательное производство), а также работу планового отдела и бухгалтерии, то при использовании процессно-ориентированного подхода на первом этапе будут внедрены все модули, отвечающие за работу этого процесса. Такой вариант позволяет сразу же выявить недостатки, допущенные на этапе планирования системы по всем участкам.

Можно сразу же проанализировать эти недостатки и внести изменения в модель-прототип системы. Если основываться на функциональном подходе, то первые результаты несоответствия межмодульного взаимодействия будут обнаружены только на этапах внедрения второго и последующих модулей системы, что, в свою очередь, может привести к значительному изменению уже внедренных модулей системы. Внесение же изменений в уже работающие модули обя- зательно повлечет за собой усложнение - повышение энтропии системы, но никак не оптимизацию их работы.

Постоянное внесение изменений в систему приведет в конечном итоге к необходимости перепроектирования системы в целом. В случае функционально ориентированного подхода это обычно выливается в реорганизацию. При использовании процессноориентированного подхода для внедрения системы появляется прекрасная возможность заняться перепроектированием системы уже на первых этапах реализации, а не применять реорганизацию на более поздних этапах проекта как некий временный вариант выхода из создавшейся ситуации.

Контроль, поддержка и оптимизация. Результатом проведения полнофункционального внедрения при процессно-ориентированном подходе является хорошо структурированная система, а также набор стандартных моделей функционирования и взаимодействия подразделений, использование которых намного упростит работы по дальнейшему развитию, модернизации, обучению и контролю системы.

Таким образом, применение процессноориентированного подхода к организации системы управления на предприятиях лесного комплекса позволит:

$>$ лучше представить систему на этапе планирования; выявить структурные и процедурные ошибки управления и выработать процедуры по их устранению;

$>$ получить наилучшие результаты на этапе внедрения, снизить трудозатраты на поддержку и развитие системы (это обусловлено хорошей структурированностью полученной системы и введением стандартов);

$>$ снизить стоимость проекта в целом и сократить сроки его внедрения.

Хочется также отметить, что внедрение системы управления на предприятиях лесного комплекса даст прекрасные результаты, такие как:

$>$ быстрая реакция на изменения окружающей среды;

$>$ заинтересованность всех сотрудников в конечном результате;

$>$ оптимальный механизм обмена информацией между функциональными подразделениями.

\section{СПИСОК ЛИТЕРАТУРЫ}

1. Шеер А. В. Бизнес-процессы. М.: ВестьМетатехнология, 1999.

2. Каменнова М., Громов А., Ферапонтов М., Шматалюк А. Моделирование бизнеса. М.: ВестьМетатехнология, 2001.

3. http://www.interface.ru Внедрение ЕRP-систем: шаг за шагом. 